\title{
Efficient calculation of critical parameters in SU(2) gauge theory it
}

\author{
J. Engels ${ }^{a}$, J. Fingberg ${ }^{b}$ and V.K. Mitrjushkin ${ }^{c, 1}$ \\ a Fakultät für Physik, Universität Bielefeld, W-4800 Bielefeld, FRG \\ b HLRZ, Forschungszentrum Jülich, Pf. 1913, W-5170 Jülich, FRG \\ c Fachbereich Physik, Humboldt-Universität, O-1040 Berlin, FRG
}

Received 28 September 1992

\begin{abstract}
We show how the critical point and the ratio $\gamma / \nu$ of critical exponents of the finite temperature deconfinement transition of SU (2) gauge theory may be determined simply from the expectation value of the square of the Polyakov loop. In a similar way we estimate the ratio $(\alpha-1) / \nu$. The method is based on a consistent application of finite size scaling theory to results obtained with the density of states technique. It may also be used in other lattice theories at second order transitions.
\end{abstract}

Finite size scaling (FSS) techniques have now become a well-established tool for the investigation of critical properties in SU(2) [1-3] and SU(3) [4$6]$ lattice gauge theories at finite temperature. Many ingenious methods have been devised using FSS theory to extract the infinite volume critical point and ratios of critical exponents from various variables. Most of these methods were invented in statistical physics and applied to high precision Monte Carlo data of Ising [7,8] and other comparatively simple models. Especially Binder's fourth-order cumulant [9] of the magnetization or the energy has become a favourite observable for the determination of the critical point. Besides that the peak positions of thermodynamic derivatives provide finite lattice transition points, which may be extrapolated by FSS formulas to the asymptotic critical point.

Both the cumulant and the susceptibility, which are the most used observables for this purpose are quantities, which involve differences of powers of directly measured observables and require thus higher statistics to reach the same accuracy. Moreover, instead of the true susceptibility a pseudosusceptibility is commonly used, where the expectation value of the mag-

\footnotetext{
Work supported by the Deutsche Forschungsgemeinschaft.

1 Permanent address: Joint Institute for Nuclear Research, 101 000 Dubna, Russian Federation.
}

netization is replaced by the one of the modulus of the magnetization.

In this letter we want to show that the FSS behaviour of the expectation value of the square of the magnetization, though it is not peaked at the transition, allows to determine the asymptotic critical point and the ratio of the critical exponents $\gamma$ and $\nu$.

We apply our idea to $\mathrm{SU}(2)$ gauge theory on $N_{\sigma}^{3} \times N_{\tau}, N_{\tau}=4$ lattices using the standard Wilson action

$S(U)=\frac{4}{g^{2}} \sum_{p}\left(1-\frac{1}{2} \operatorname{Tr} U_{p}\right)$,

where $U_{p}$ is the product of link operators around a plaquette. The number of lattice points in the space (time) direction $N_{\sigma(\tau)}$ and the lattice spacing $a$ fix the volume and temperature as

$V=\left(N_{\sigma} a\right)^{3}, \quad T=1 / N_{\tau} a$.

On an infinite volume lattice the order parameter of magnetization for the deconfinement transition is the expectation value of the Polyakov loop

$L(\boldsymbol{x})=\frac{1}{2} \operatorname{Tr} \prod_{\tau=1}^{N_{\tau}} U_{\tau, \boldsymbol{x} ; 4}$,

or else, that of its lattice average

$L=\frac{1}{N_{\sigma}^{3}} \sum_{x} L(\boldsymbol{x})$, 
where $U_{x ; 4}$ are the SU(2) link matrices of four-position $x$ in time direction.

Since, due to system flips between the two ordered states in finite lattices the expectation value $\langle L\rangle$ is always zero, the true susceptibility

$\chi=N_{\sigma}^{3}\left(\left\langle L^{2}\right\rangle-\langle L\rangle^{2}\right)$,

reduces there to

$\chi_{\nu}=N_{\sigma}^{3}\left\langle L^{2}\right\rangle$.

The quantity $\chi_{\nu}$ is monotonically rising as a function of $\beta=4 / g^{2}$ or the temperature $T$. At first sight there is no hint of the transition point. However, below the critical point

$\chi_{v}=\chi$,

and we expect therefore $\chi_{v}$ to have the FSS behaviour of the susceptibility [1] for $T \lesssim T_{\mathrm{c}}$

$\chi_{\nu}=N_{\sigma}^{\gamma / \nu} Q_{\chi}\left(x N_{\sigma}^{1 / \nu}, x_{i} N_{\sigma}^{y_{i}}\right)$.

Here

$x=\frac{T-T_{\mathrm{c}, \infty}}{T_{\mathrm{c}, \infty}}$ or $\quad x=\frac{4 / g^{2}-4 / g_{\mathrm{c}, \infty}^{2}}{4 / g_{\mathrm{c}, \infty}^{2}}$,

is the reduced temperature and $Q_{\chi}$ is a scaling function with possible additional dependencies on irrelevant scaling fields $x_{i}$ and exponents $y_{i}<0$ leading to correction-to-scaling terms.

Taking into account only the largest irrelevant exponent $y_{1}=-\omega$ and expanding the scaling function $Q_{x}$ around $x=0$ one arrives at

$\chi_{\nu}=N_{\sigma}^{\gamma / \nu}\left[c_{0}+\left(c_{1}+c_{2} N_{\sigma}^{-\omega}\right) x N_{\sigma}^{1 / \nu}+c_{3} N_{\sigma}^{-\omega}\right]$.

If we take now the logarithm of the last equation at $x=0$ we find

$$
\begin{aligned}
& \ln \chi_{v}=\ln \left(N_{\sigma}^{3}\left\langle L^{2}\right\rangle\right) \\
& =\ln c_{0}+\frac{\gamma}{\nu} \ln N_{\sigma}+\frac{c_{3}}{c_{0}} N_{\sigma}^{-\omega}+\ldots,
\end{aligned}
$$

i.e., apart from probably small correction-to-scaling terms $(\omega \approx 1[3])$, we have a linear dependence on $\ln N_{\sigma}$ with slope $\gamma / \nu$, whereas for $x \neq 0$ the $N_{\sigma}$-behaviour is drastically changed due to the presence of $x N_{\sigma}^{1 / \nu}$-terms $(1 / \nu \approx 1.59[8])$. We shall take advantage of this fact to determine the critical point as that $\beta$-value where a linear fit of $\ln \chi_{\nu}$ as a function of $\ln N_{\sigma}$ has the least minimal $\chi^{2}$ and/or highest goodness of fit. In addition we obtain then the value of $\gamma / \nu$ from the slope at the critical point.

The SU(2) Monte Carlo data, which we want to use here for a demonstration of the above method were computed on $N_{\sigma}^{3} \times N_{\tau}$ lattices with $N_{\sigma}=8,12$, 18,26 and $N_{\tau}=4$ and have already been reported on in refs. [1,3], apart from one new point. Though these data were taken at many (except on the largest lattice) $\beta$-values in the neighbourhood of the critical point this is not sufficient for a systematic search for the asymptotic critical point. The necessary interpolation may, however, be performed with the density of states method (DSM) [10]. Following the same lines as in ref. [3], we have reevaluated the data in the very close vicinity $(2.2980 \leqslant \beta \leqslant 2.3005)$ of the transition. In fig. 1 we show $\chi_{v}$ as a function of $\beta$ for the different lattices. The error corridors were calculated with the jackknife method. The results from the $N_{\sigma}=26$ lattice have the largest errors. There only four overlapping histograms were available from refs. $[1,3]$. To improve this situation and to test the stability of the DSM interpolation we calculated one new point at $\beta=2.2988$ on the $26^{3} \times 4$ lattice with a 9 times higher statistics as compared to the point at $\beta=2.30$. We observed no change in the DSM interpolation of

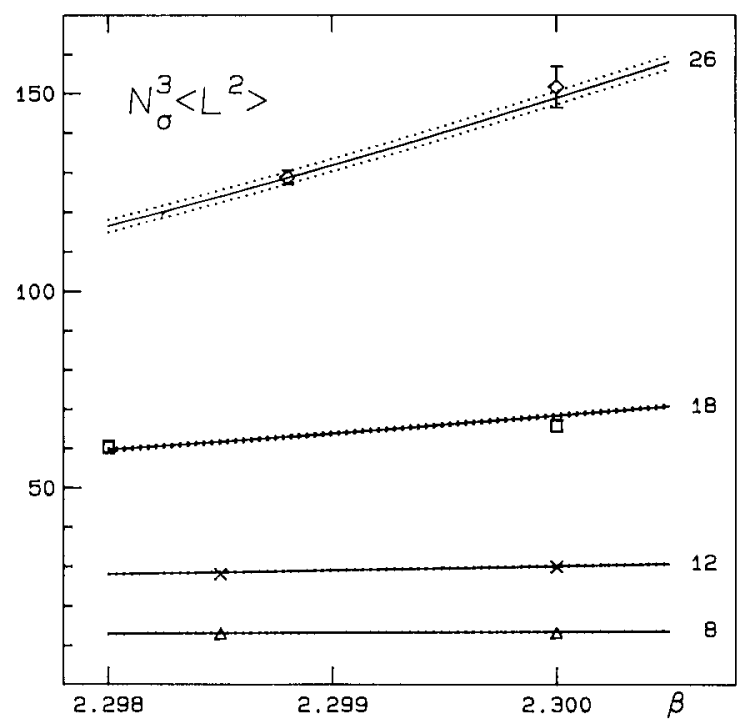

Fig. 1. The expectation value of the square of the lattice averaged Polyakov loop $N_{\sigma}^{3}$ as a function of $\beta=4 / g^{2}$ for $N_{\sigma}=8,12,18,26$ and $N_{\mathrm{\tau}}=4$. The solid lines were calculated from the DSM, the dotted lines indicate the errors. 
$\chi_{v}$, however the error corridor decreased by $50 \%$. The data points in fig. 1 are the directly measured quantities and indicate the $\beta$-values of the corresponding histograms falling into our $\beta$-interval.

In fig. 2 we present the result of a linear $\chi^{2}$ fit of $\ln \chi_{v}$ as a function of $\ln N_{\sigma}$. At each $\beta$-value we have determined the minimal $\chi^{2} / N_{\mathrm{f}}$, with $N_{\mathrm{f}}=2$ the number of degrees of freedom. We see that there is a unique $\beta$-value, where the four data points for $N_{\sigma}=8$, $12,18,26$ lie on a perfect straight line. We consider this value,

$\beta_{\min }=2.2988(1)$,

to be a very good estimate of the infinite volume critical point. Indeed it is in excellent agreement with the best determination from the cumulant [3]

$\beta_{\mathrm{c}, \infty}=2.2986(6)$,

and, as is evident from fig. $2, \beta_{\min }$ is fixed by the data with extreme precision. The error in eq. (12) was estimated from the change in $\beta_{\min }$ which was induced due to the inclusion of our high statistics point on the largest lattice. The slope of each linear fit is compared to $\gamma / \nu$ from the three-dimensional Ising model. That model and SU(2) gauge theory are supposed to

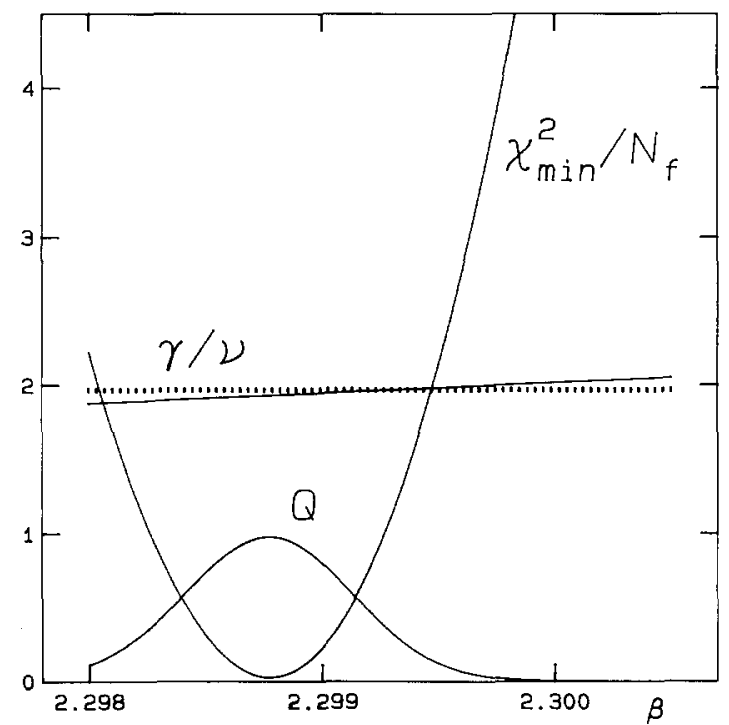

Fig. 2. The minimal $\chi^{2}$ per degree of freedom if at each $\beta$ a linear fit of the logarithm of $\chi_{v}=N_{\sigma}^{3}\left\langle L^{2}\right\rangle$ as a function of $\ln N_{\sigma}$ is performed; $Q$ is the goodness of fit. The ratio $\gamma / \nu$ is the slope of the fit; the dotted line the $3 \mathrm{D}$ Ising model value. be in the same universality class [11] and therefore to have coinciding critical exponents.

The slope of the linear fit which we find at $\beta_{\min }$ is

$\gamma / \nu=1.931(15)$,

i.e. less than $2 \%$ different from the value

$\gamma / \nu_{\mathrm{I}}=1.970(11)$,

of the three-dimensional Ising model [8]. The error in eq. (14) comes from the error in $\beta_{\min }$ and the error of the slope from the linear fit. In fig. 3 we show the fits at $\beta_{\min }$ with these two slopes, respectively. We observe only for the highest $\ln N_{\sigma}\left(N_{\sigma}=26\right)$ a slight difference.

The critical exponent $\alpha$ of the specific heat is difficult to determine directly. From the hyperscaling relation

$\alpha=2-d \nu$,

one estimates $\alpha \approx 0.11$. The non-singular parts in the specific heat and the energy density are therefore dominating. This leads to a modified scaling ansatz for the energy density,

$\epsilon=\epsilon_{\text {regular }}+N_{\sigma}^{(\alpha-1) / \nu} Q_{\epsilon}\left(x N_{\sigma}^{1 / \nu}\right)$,

where we have already neglected the irrelevant scal-

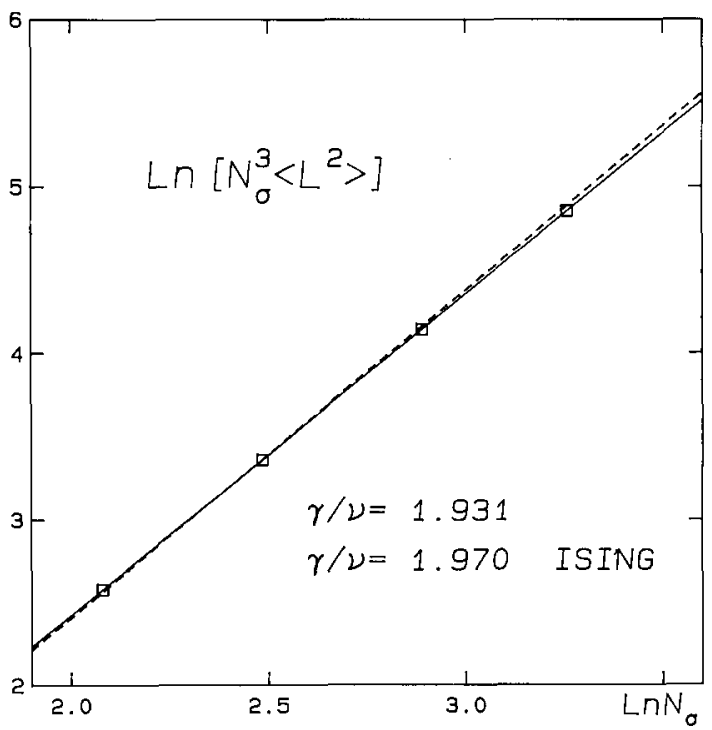

Fig. 3. The best linear fit at the minimum of $\chi^{2}$, i.e. at $\beta_{\min }$ (solid line) and a fit with the slope fixed to the $3 D$ Ising model value (dashed line) 
ing fields. The regular part of the energy density is assumed to be, up to exponentially damped contributions, independent of $N_{\sigma}$. The respective singular part of the pressure is proportional to $N_{\sigma}^{-d}, d=3$ and therefore also much less size dependent than the singular part of the energy. Linear combinations of the energy and the pressure $P$ are then generally expected to behave like

$c_{1}+N_{\sigma}^{(\alpha-1) / v} c_{2}, \quad c_{1,2}=$ const.

at $x=0$. Since apart from only $g^{2}$ but not $N_{\sigma}$ dependent factors [12]

$P_{\sigma}-P_{\tau} \sim(\epsilon+P) / T^{4}$,

and

$P_{\sigma}+P_{\tau} \sim(\epsilon-3 P) / T^{4}$,

where $P_{\sigma}$ and $P_{\tau}$ are the expectation values of the space and time plaquettes, one may use the last three relations to determine $(\alpha-1) / \nu$ in a similar manner as we have done it before to obtain $\gamma / \nu$. There are, however, some modifications. First, due to the regular term represented by the constant $c_{1}$ we cannot just fit the logarithm to a straight line in $\ln N_{\sigma}$. Instead we shall use as variable $N_{\sigma}^{(\alpha-1) / \nu}$, where we prefix the exponent and then look for the best linear approximation at each $\beta$-value. As a consequence we have a three parameter fit for $c_{1}, c_{2}$ and the exponent $(\alpha-1) / \nu$. The measured values for $P_{\sigma}-P_{\tau}$ and $P_{\sigma}+P_{\tau}$ were again interpolated with the density of states method in the close vicinity of the deconfinement transition. Quite similarly as for the energy of the three-dimensional Ising model [8] we observed noticeable systematic errors in the interpolation results for $P_{\sigma}+P_{\tau}$. This was not so pronounced in the case of the plaquette difference $P_{\sigma}-P_{\tau}$. We have therefore only evaluated the latter quantity. In contrast to the DSM interpolation for $\left\langle L^{2}\right\rangle$ on the largest lattice, which was unaffected by the inclusion of the additional histogram, we found a considerable change in the DSM interpolation leading to a steeper slope of the plaquette difference. The resulting critical exponent ratios which we shall calculate below will therefore have to be taken with some care.

A plot of the DSM results for $P_{\sigma}-P_{\tau}$ versus $\beta$ for the different lattice sizes is shown in fig. 4 . To take into account the systematic errors due to the DSM interpolation and the uncertainties coming from pos-

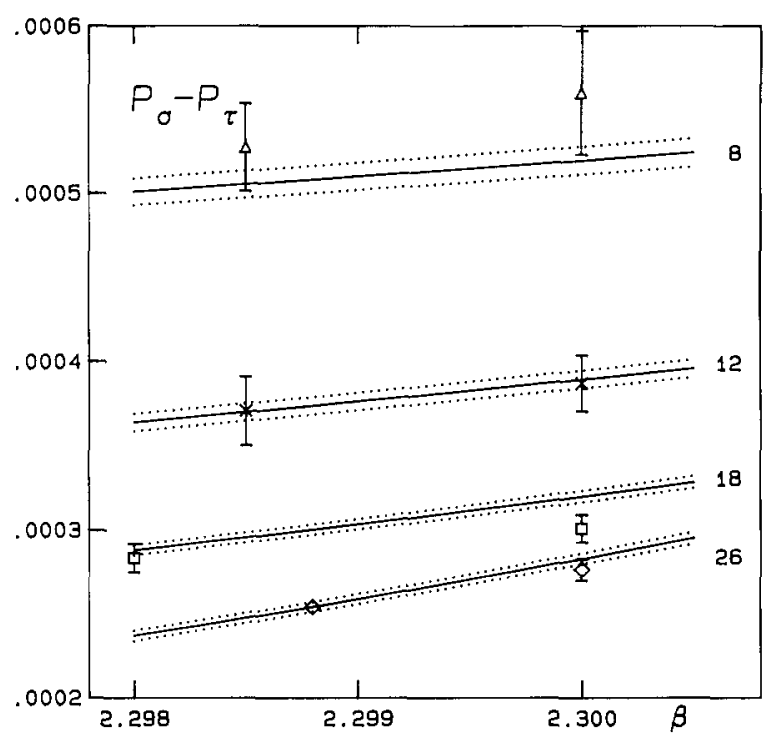

Fig. 4. The difference of the space and time plaquette expectation values as obtained from the DSM for the same lattices as in fig. 1.

sibly too low statistics we proceed as follows. Since the interpolations for $N_{\sigma}=18$ and 26 are probably the least reliable, we make three different fits: one where we discard the $N_{\sigma}=26$ data, one where the $N_{\sigma}=18$ data are omitted - in these cases we have three parameters and three points and therefore exact solutions - and a fit with all data. The corresponding solutions for the exponent $(\alpha-1) / \nu$ are compared in fig. 5 to the Ising value [8] obtained from the hyperscaling relation

$(\alpha-1) / \nu=1 / \nu-d \approx-1.41$.

Averaging the three solutions at $\beta_{\min }$ we find

$(\alpha-1) / \nu=-1.36(14)$.

In addition to the ratio of critical exponents we obtain information on the size of the regular part at the transition point. This is of importance for theoretical models of the deconfinement transition. The constant $c_{1}$ from the fit is $1.93(19) \times 10^{-4}$, or expressed with the energy density and pressure

$(\epsilon+P) / T_{\text {regular }}^{4}=0.38(4)$.

Here, the main contribution will be that of the energy density, because the size of the total pressure is only about $7 \%$ of the size of the regular sum in eq. (23). 


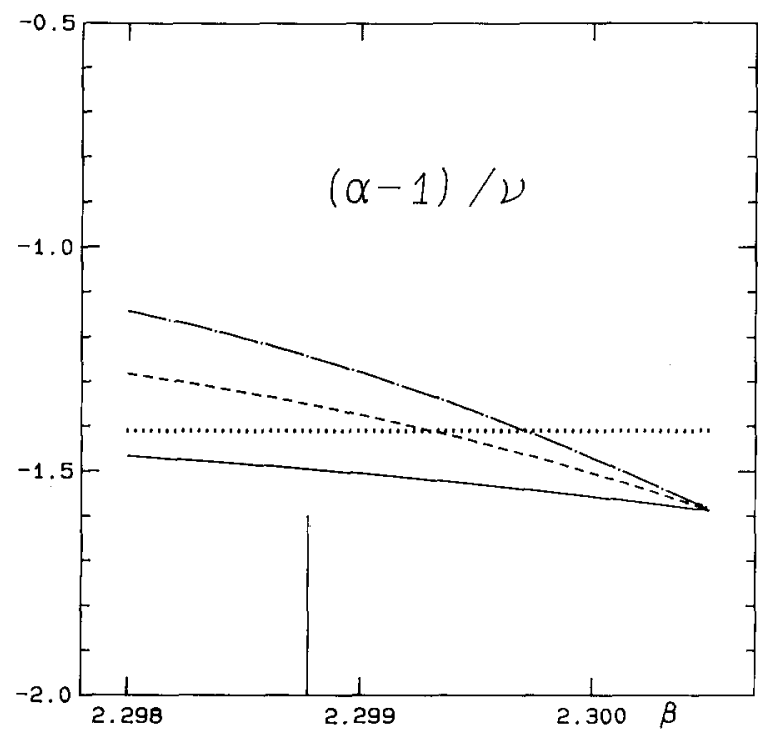

Fig. 5. The ratio $(\alpha-1) / \nu$ obtained from linear fits to $P_{\sigma}-P_{\tau}$ with the ansatz eq. (24), using only the $N_{\sigma}=8,12,18$ data (solid line), only the $N_{\sigma}=8,12,26$ data (dashed line) and from all lattices (dot-dashed line). The points indicate the 3D Ising model value from the hyperscaling relation.

We are indebted to the computer center of the University of Cologne, where we calculated our additional high statistics histogram on the largest lattice.

\section{References}

[1] J. Engels, J. Fingberg and M. Weber, Nucl. Phys. B 332 (1990) 737.

[2] B. Berg and A. Billoire, Phys. Rev. D 40 (1989) 550.

[3] J. Engels, J. Fingberg and D.E. Miller, Nucl. Phys. B 387 (1992) 501.

[4] M. Fukugita, M. Okawa and A. Ukawa, Phys. Rev. Lett. 63 (1989) 1768; Nucl. Phys. B 337 (1990) 181.

[5] N.A. Alves, B.A. Berg and S. Sanielevici, Phys. Rev. Lett. 64 (1990) 3107; Florida State University preprint FSUSCRI-91-93.

[6] Y. Iwasaki et al., Phys. Rev. Lett. 67 (1991) 3343; preprint UTHEP-237 (1992).

[7] M.N. Barber, R.B. Pearson, D. Toussaint and J.L. Richardson, Phys. Rev. B 32 (1985) 1720.

[8] A.M. Ferrenberg and D.P. Landau, Phys. Rev. B 44 (1991) 5081.

[9] K. Binder, Z. Phys. B 43 (1981) 119.

[10] G. Bhanot, S. Black, P. Carter and R. Salvador, Phys. Lett. B 183 (1986) 331;

G. Bhanot, K. Bitar, S. Black, P. Carter and R. Salvador, Phys. Lett. B 187 (1987) 381;

G. Bhanot, K. Bitar and R. Salvador, Phys. Lett. B 188 (1987) 246;

M. Falconi, E. Marinari, M.L. Paciello, G. Parisi and B. Taglienti, Phys. Lett. B 108 (1982) 331;

E. Marinari, Nucl. Phys. B 235 (1984) 123;

A.M. Ferrenberg and R.H. Swendsen, Phys. Rev. Lett. 61 (1988) 2635; 63 (1989) 1195.

[11] B. Svetitsky and G. Yaffe, Nucl. Phys. B 210 [FS6] (1982) 423.

[12] J. Engels, Nucl. Phys. B (Proc. Suppl.) 332 (1990) 325. 